\title{
TEMPERATURE DISTRIBUTION OF A TOKAMAK WITH A CONSTANT HEAT CONDUCTIVITY
}

\author{
S. Yoshikawa \\ Princeton Plasma Physics Laboratory, Princeton University \\ Princeton, NJ 08543, USA
}

\begin{abstract}
PPPL- -2666
Abstract

DE90 010344

An analytical expression of the detached plasma radius in an ohmically heated tokamak plasma was obtained. The assumption was made that the (anomalous) heat conductivity is constant and independent of minor radius, $r$. The resultant nonlinear differential equation has a universal solution for the plasma temperature as a function of $r$. The shape is very similar to the so-called profile consistency model. As the average plasma density increases, the tokamak plasma which is first attached to either limiter or divertor detaches itself from the limiter and forms a toroidal plasma whose boundary is clearly marked by a radiative boundary layer where the power input to the plasma is radiated away. As the plasma density increases, the radius of the plasma shrinks until the surface safety factor becomes less that -2 , whereupon the plasma disruption starts. The density limit calculated by this model agrees with the experimental observation.
\end{abstract}

\section{DISCLAIMER}

Thepared as an account of wurk sponsored by an agency of the United States This report weither the United States Government nor any agency thereol. nor any of ther Neress or implied, or assumes any legal hinblity, product, or cmployees, makes any warrantex, ex uselulness of any information, appated rights. Referbility for the accuracy, completents, its use would not infringe pricaty by trade name, trademark, process disclosed, or represents that ils product, process, or service by trade endorsement, recom. ence herein to any specilic comnereial pecessarily constitute or imply ils endereot. The views manufacturet, or otherwise does United States Government or any agency thect those of the mendation, of favoring by the Unined herein do not

and opinions of authors expressed hency theresf.

United Stales Government or any agency there

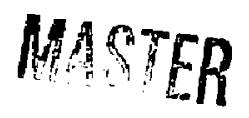




\section{Introduction}

Both density and temperature distributions in a tokamak plasma can be determined numerically, if the boundary conditions, the transport coefficients, radiation power loss and power input are known. In practice, these quantities are, at best, imprecisely known. Hence, prediction of temperature distribution, for example, depends heavily on numerically adjusted plasma parameters. Because there are a number of adjustable parameters, the numerical results can reasonably explain the actual experimental results for the wide range in densities and temperatures of tokamak plasmas. However, several entirely different sets of assumptions can almost equally well fit the experimental data.

In view of the complexities of the plasma behavior, some may question the usefulness of an (of necessity, simplified) analytical attempt to understand the behavior of iokamak temperature distributions. The absence of analytical solutions, however, leads often to the misunderstandings of the proper behavior of tokamak plasmas. For example, there are many reports of the detached plasmas in the literature: but sometimes, the reports claim that the minor radii of the plasmas could be defined by the magnetohydro. iynamical (MHD) consideration alone. ${ }^{1}$

To explain the experiments, ${ }^{2}$ that the transport equations alone can determine the minor radii of detached plasmas, subject to the condition that the safety factor, $q$ at the plasma surface must be $\geq 2 .^{3}$ The theory nor experiments are not complete in the sense that the plasma density profile was assumed to be given. Ideally the densiiy profiles must be determined by plasma parameters along with the proper boundary conditions. However, our knowledge is not advanced enough to enable us to predict the density profiles, unambiguously.

Therefore, we shall assume that the plasma density profile is of the form, $n_{\circ} g(r)$ and $g(r)$ is independent of plasma conditions, in particular, $g(r)$ is the same whether the plasma is detached or not. Also we assume that at the limiter or divertor edge, $g(r)$ is non-zero, say $>1 / 4$. (The precise number is not important). 
In order to get concrete analytical results, we assume that the variables depend on only minor radius, $r$, hence time-independent and one-dimensional. We assume that the heat conductivity, $K$, is constant independent of $r$ and the plasma is represented by one temperature $T(r)=T_{e}(r)=T_{i}(r)$. For simplicity, $Z_{\text {eff }}=$ const. However, we retain the atomic radiation $S\left(\right.$ watts $/ \mathrm{m}^{3}$ ) due to the excitation and recombination of impurity ions. The function $S$ is non-zero only when $T \leq T_{b}$. In this paper, to be consistent with the realistic plasma, $\mathrm{S} \approx 0$ if $\mathrm{T} \leq \mathrm{T}_{\mathrm{C}}$. Typically $\mathrm{T}_{\mathrm{b}} \approx 100$ ev, $T_{c} \approx 5$ ev. We restrict ourselves to the ohmic heating case.

It may be argued that this model is too simplified. However, we must note that more complications can only be introduced by the additional assumptions which are not usually deduced from the theory alone. (For example, recycling coefficient.) That is, the additional refinement is achieved only by the empirical (numerical) fit. (The $\mathrm{T}=\mathrm{T}_{\mathrm{e}}=\mathrm{T}_{\mathrm{i}}$ assumption can certainly be improved upon, but the refinement will not lead to a qualitatively different result.)

\section{Solution}

If the plasma density is low or the plasma impurity is very small, the radiative heat loss is negligible, even if $\mathrm{T}<\mathrm{Tb}$. Then, within $\mathrm{r} \leq \mathrm{a}$, ( a being the limiter or diverter radius), the heat conduction must balance the ohmic heating rate $\sigma(T) E^{2}$, where $\sigma$ is the plasma electrical conductivity and $E$ is the strength of the electric field parallel to the magnetic field, which is independent of $r$. The equation is then in a dimensionless form

$$
\frac{1}{\mathrm{x}} \frac{\mathrm{d}}{\mathrm{dx}} \times \frac{\mathrm{dY}}{\mathrm{dx}}+\lambda \mathrm{Y}^{3 / 2}=0
$$

with the eigenvalue

$$
\lambda=\frac{\sigma(0) E^{2}}{T_{0} K} a^{2} .
$$

Here, 


$$
\begin{aligned}
& X=\frac{\tau}{a}, \\
& Y=\frac{T(\tau)}{T_{0}}, Y(0)=1
\end{aligned}
$$

and $\sigma(0)$ is the conductivity at $r=0$. The boundary condition of Eq. (1) is satisfied by

$$
-\left.\frac{1}{Y} \frac{d Y}{d x}\right|_{X=1}=Q\left(\frac{a}{\lambda_{s}}\right)
$$

Here $\lambda s$ is the scrape-off layer length, first discussed in Ref. 4 for toroidal, fully ionized plasmas. (Earlier, Simon ${ }^{5}$ discussed for a weakly ionized plasma.) In modern-day tokamaks $\lambda_{s} / a \ll 1$, except for special cases. Hence $Y$ can be safely assumed to be 0 at $x=1$. Then Eq. (1) yields a universe curve of $\checkmark$ with $\lambda$ approximately 7.0 (Fig. 1). In Fig. 1, the original profile consistency prediction by Coppi 6 is also shown. Since $\sigma(0)$ is proportional to $\mathrm{T}_{\mathrm{O}} 3 / 2$, Eq. (2) thus determines the total current for a given $\mathrm{E}$. Therefore, the temperature of the plasma can be predicted.

Now if the limiter temperature, $T_{0} Y(1)$, becomes less than $-100 \mathrm{eV}$, (here $\mathrm{T}_{\mathrm{O}}$ $>100 \mathrm{eV}$, then $T_{0} Y(1)$ could be $100 \mathrm{eV}$ even though $Y(1) \ll 1$ ), the atomic radiation from the impurity ions (such as $\mathrm{CV+}$ ) becomes significant. Then the condition is set for the appearance of the detached plasmas.

If the total radiative power loss is less than the total ohmic heating power input as assumed, the tokamak plasma is still attached to the limiter. (Incidentally if we allow the heat conductivity to be a function of both radius and temperature, there could be two solutions of Eq. (1) under two boundary conditions with the limiter temperature one over $100 \mathrm{eV}$ and another less than $100 \mathrm{eV}$. That may explain the difference between $\mathrm{H}$-mode arı L-mode.)

If the impurity content is high, the plasma detaches itself from the limiter and the radiative boundary layer develops. In a usual tokamak, in the layer, the ohmic 
heating power input is negligible compared with the radiative power loss, $S$. Hence in the radiative layer, we may write

$$
\kappa \frac{1}{\mathrm{r}} \frac{\mathrm{d}}{\mathrm{dr}} \mathrm{r} \frac{\mathrm{dT}}{\mathrm{dr}}=\mathrm{S} \quad 5 \mathrm{ev}<\mathrm{T}<100 \mathrm{eV}
$$

Usually $S$ is large enough so that the layer thickness is small compared with the limiter radius a. Then Eq. (6) can be solved to yield

$$
T=T_{b}-A\left(r-a_{d}\right)+\frac{1 S}{2 K}\left(r-a_{d}\right)^{2}
$$

Here $a_{d}$ is the detached plasma radius and $T_{b}$ is the temperature at which the radiation becomes significant $(\sim 100 \mathrm{eV})$. We assumed that $S$ is constant.

Within $:<\mathrm{a}_{\mathrm{d}}$, Eq. (1) applies with the radius $\mathrm{r}$ normalized by $\mathrm{a}_{\mathrm{d}}$. That is $\mathrm{x}=$ $r / a_{d}$. Now at $r=a_{d}$, the solution (7) must smoothly connect the solution of Eq. (1). (Otherwise there is discontinuity in the heat flux.) Therefore, $\mathrm{A}$ is determined at the boundary condition at $\mathrm{r}=\mathrm{a}_{\mathrm{d}}$. At the point where the radiation is no longer important (at $\mathrm{T}_{\mathrm{C}} \approx 5 \mathrm{eV}$ ), however, the temperature gradient must almost vanish. For otherwise, the heat flux at that point could not be transported away. But, that is highly unlikely. Thus $d T / d r \approx 0$ at the outside of the boundary layer imposes the condition on $A$, which, in turn, determines the detached plasma radius, $a_{d}$. The radius is given as 3

$$
\mathrm{a}_{\mathrm{d}}^{4}=\frac{\lambda \mathrm{T}_{\mathrm{o}} \mathrm{I}^{2}}{8 \mathrm{\pi}^{2} \mathrm{ST} \mathrm{T}_{\mathrm{b}} \sigma(0)}, \quad \lambda \approx 7.0
$$

if $K$ is eliminated by means of Eq. (2). Here $I$ is the total plasma toroidal current. Various other expressions of ad are possible, depending upon what variables are to be eliminated. (The equation (7) has an important physical significance. As $A$ is determined by the boundary condition from the solution of Eq. (1), the distance ( $\left.r-a_{d}\right)$ at which $T$ reaches zero is much smaller than the distance between the limiter radius, $a_{L}$, and $a_{d}$. Hence the boundary layer, $\xi_{b}$, is much less than $a_{L}-a_{d}$. It follows that between $r=a_{d}+\xi_{b}$ and $r=a_{L}$, the temperature must be less than $T \leq T_{c}$. This result is 
completely in agreement with the experiments.2,7)

Recently the analysis was extended including the auxiliary heating. 7,8

Physically, the detached plasma radius is determined by the power deposited in the plasma which is large enough to maintain the radiative boundary layer. The layer thickness, $\xi_{\mathrm{b}}$, is determined as

$$
\xi_{\mathrm{b}}=\sqrt{\frac{2 \kappa T_{b}}{S}}
$$

from Eq. (7). It follows that the plasma radius is datermined from the condition that the power deposited in the plasma, $\mathrm{P}$, must be balanced by the total radiation

$$
4 \pi^{2} \operatorname{RaD} E_{b} S=P
$$

Here $R$ is the major radius. Thus if $P$ is increased by the auxiliary power, $a_{d}$ is expected to increase for a constani $k$ (or if $k \propto P^{1 / 2}$ ).

A cursory look into the stability of this equilibrium is that the system is stable. That is, if the plasma radius is slightly larger than ad, the plasma size shrinks (and vice versa).

III. Discussions

A. The power to the limiter or the clivertor

The power flow to the limiter or the divertor should become practically zero as soon as the plasma detaches itself, unless the plasma MHD equilibrium is lost (as in Marfe). This conclusion comes quite naturally from the discussions given after Eq. (7). Hence radiative layer which locates less than the limiter radius, $a_{r}$ cannot radiate away only a fraction of the input power (minus the charge exchange power loss, if any). Thus, the radiative layer, sometimes called the virtual limiter, cannot be located at arbitrary minor radius. That is the MHD stability consideration alone cannot 
determine the detached plasma radius.

B. Plasma disruption

The expression (8) can be rewritten as

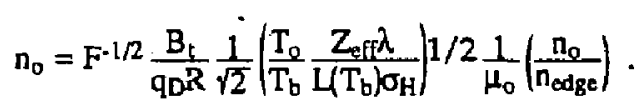

Here $F$ is the ratio of impurity density over the electron density at the plasma edge, $q_{D}$ is the safety factor also at the edge, $Z_{\text {eff }}$ is the effective ion charge number at the plasma center, $\mathrm{L}\left(\mathrm{T}_{\mathrm{b}}\right)$ is the radiation intensity, $\sim 10^{-32}$ watts $\cdot \mathrm{m}^{3}$, (for carbon or oxygen) $\sigma_{H}$ is the hydrogen electrical conductivity at the center, $\mu_{\mathrm{O}}$ is the vacuum magnetic permeability, $n_{0}$ is the center density, and $n_{\text {edge }}$ is the edge density.

From the MHD kirk/tearing instability consideration, if $q_{D}$ is less than 2, the plasma should become MHD unstable. Hence if the density in Eq. (11) becomes greater than the value given at $\mathrm{q}_{D}=2$, the plasma is expected to disrupt. For several parameters, the tinreshold density for theoretisally predicted value is given in Table I. The peak temperature, $T_{0}$ (the dependence of density is $T_{0}{ }^{-1 / 4}$ ), was used, instead of the heat conductivity. It is justified, because the heat conductivity cannot be given a priori. Since major tokamaks such as JET, TFTR, and JT-60 have the ohmic heating electron temperatures between 2 to $6 \mathrm{keV}$, the Table I values indicate that the value $n_{0} R / B_{t}$ (in unit of $10^{20}$ ) to be approximately 0.8 to 1.8 . This value is consistent with the reported values of $\overline{\mathrm{nR}} / \mathrm{B}_{\mathfrak{l}}$ by the JET team, which is about 0.5 .9

The pellet injection tends to increase more of the central density and less of the edge density. Therefore, the density limit for the disruption could be increased. This theoretical result agrees with the experimental result qualitatively.

Plasma disruptions of tokamak plasmas can be induced by various means. For example, if the total current is increased for a fixed parameter, the surface safety parameter $q$ can become less than -2 and the disruption commences. Also if the sudrien plasma heating redistributes the plasma current, the disruption results. 
Finally the feedback stabilization mechanism of the plasma equilibrium will lead to the disruption.

If the plasma density is suddenly increased, however, the disruption takes place. The disruption is usually so sudden, that adequate study of the disruption is hitherto unavailable. The model described here adequately explains, how the disruption takes place if the plasma density is increased gradually. When the sudden plasma density increase takes place, it is quite possible that the violent redistribution of the current distribution takes place. That presumably will trigger the plasma disruption.

\section{Plasma Density Limit}

From the design point of view of a high temperature plasma confinement device for the controlled thermonuclear reaction, the density reeds to be as high as possible within the MHD (interchange) stability limit. According to the model presented here, the density limit follows what was predicted by Murakami, et al. ${ }^{10.11}$ The Murakami limit could be prevented by keeping the detached plasma radius large. This can be accomplished (i) by reducing the impurity radiation, (ii) by increasing the total power input, or (iii) by peaking the density profile. Qualitatively experiments support these conclusions.

\section{Conclusions}

It is expected that conclusions arrived at in Sec. 3 will not be seriously modified by the future refinement of the theoretical approaches, as the conclusions in Sec. 3 can be also arrived at with less restrictive condition than the heat conductivity being constant.

For future studies of the plasma confinement, it is necessary that the results are interpreted depending whether the plasma is attached or detached. The discrimination between the two should be relatively easy by measuring the heat flux to the limiter or the divertor or by analyzing bolometer signals located at the vacuum 
chamber wall.

\section{Acknowledgment}

This work supported by United States Department of Energy Contract No. DE-ACO2$76 \mathrm{CHO} 073$. 


\section{References}

${ }^{1} \mathrm{H}$. Soltwisch, W. Stodiek, J. Manickam, and J. Schluter, in Proceedings of the 13tii International Conference Plasma Physics ard Controlled Nuclear Fusion Research 1985, Kyoto (IAEA, Vienna, 1986) Vol. 1, p. 263.

2J.D. Strachan, E.P. Boody, C. Bush, B. Grek, R.J. Hawryluk, D. Hısifetz, H.W. Hendel, K.W. Hill, D.W. Johnson, A.T. Ramsey, S. Sesnic, J.F. Schivell, M. Shimada, G. Taylor, and M.C. Zarnstoff in Proceedings of the 12th European Conference on Cor:rolled Fusion and Plasma Physics, (European Physical Society, Geneva, 1985) Pt. 1, p. 339.

${ }^{3}$ S. Yoshikawa and M. Chance, Princeton Plasma Physics Laboratory Report No. PPPL2362 (1986).

4S. Yoshikawa, W.L. Harries, and R.M. Sinclair, Phys. Fluids $\underline{6} 1506$ (1963).

5A. Simon, in Proceedings of the 2nd International Conference of Peaceful Uses of Atomic Energy, (IAEA, Geneva, 1958) 32, 342. Also see S. Yoshikawa, Ph.D. Thesis (Massachusetts Institute of Technology, Cambridge, Massachusetts, 1961) Chapter 8.

6B. Coppi, Comments on Plasma Physics and Controlled Fusion, 5, 261 (1980).

7C.E. Bush, J.D. Strachan, J. Schivell, S.S. Medley, G. Taylor, H.H. Towner, R.M. Wieland, M.G. Bell, P.C. Efthimion, B. Grek, D.W. Johnson, D.K. Mansfield, D.H. McNeill, W. Morris, D. Mueller, H. Park, and S. Yoshikawa, Bull. Am. Phys. Soc. 32 1927 (1987).

8P. Guzdar and C.S. Liu. Phys. Fluids (in press).

${ }^{9}$ The JET Team, in Proceedings of the 13th International Conference Plasma Physics and Controlled Nuclear Fusion Research 1986, Kyoto, (IAEA, Vienna, 1987) Vol. I, p. 31 .

10M. Murakami, J.D. Callen, and L.A. Berry, Nucl. Fusion 16, 347 (1976). 
11D. stotler, Fhys. Fluids, 31,3713 (9988). 


\begin{tabular}{|c|c|c|c|c|}
\hline$F$ & 0.05 & 0.02 & 0.01 & 0.01 \\
\hline$T_{0}(e v)$ & 3000 & 3000 & 4000 & 2000 \\
\hline $\mathcal{Z}_{\text {eff }}$ & 4 & 2.5 & 1.5 & 1.5 \\
\hline$n_{0} / n_{\text {edge }}$ & 3 & 3 & 5 & 2.5 \\
\hline$L\left(w \cdot m^{3}\right)$ & $1.0 \mathrm{E}-32$ & $1.0 \mathrm{E}-32$ & $1.0 \mathrm{E}-32$ & $1.0 \mathrm{E}-32$ \\
\hline $\mathrm{T}_{b}(\mathrm{ev})$ & 200 & 100 & 100 & 100 \\
\hline noR/Bt & 0.87 & 1.09 & 1.85 & 1.10 \\
\hline \multicolumn{5}{|l|}{$\left(10^{20} \mathrm{~m}^{-2} / \mathrm{T}\right)$} \\
\hline $\begin{array}{l}\mathrm{n}_{\mathrm{o}}\left(10^{20} \mathrm{~m}^{-3}\right) \\
\text { (for } B=5 \mathrm{~T}, \mathrm{R}=2\end{array}$ & 1.75 & 2.18 & 3.71 & 2.20 \\
\hline
\end{tabular}

Table I. Tokamak Density Limit. Note that the table is universal. That is, the limit is independant of the total plasma current or the limiter minor radius, if the limiter $\mathrm{q}$ is $10>q>2$. The predicted central density, $n_{0}$, is tabulated. 
Figure Caption

Fig. 1 Comparison of constant heat conductivity solution (solid line) compared with profile consistency solution, $\exp \left(-2 x^{2}\right)$ (dotted line) 


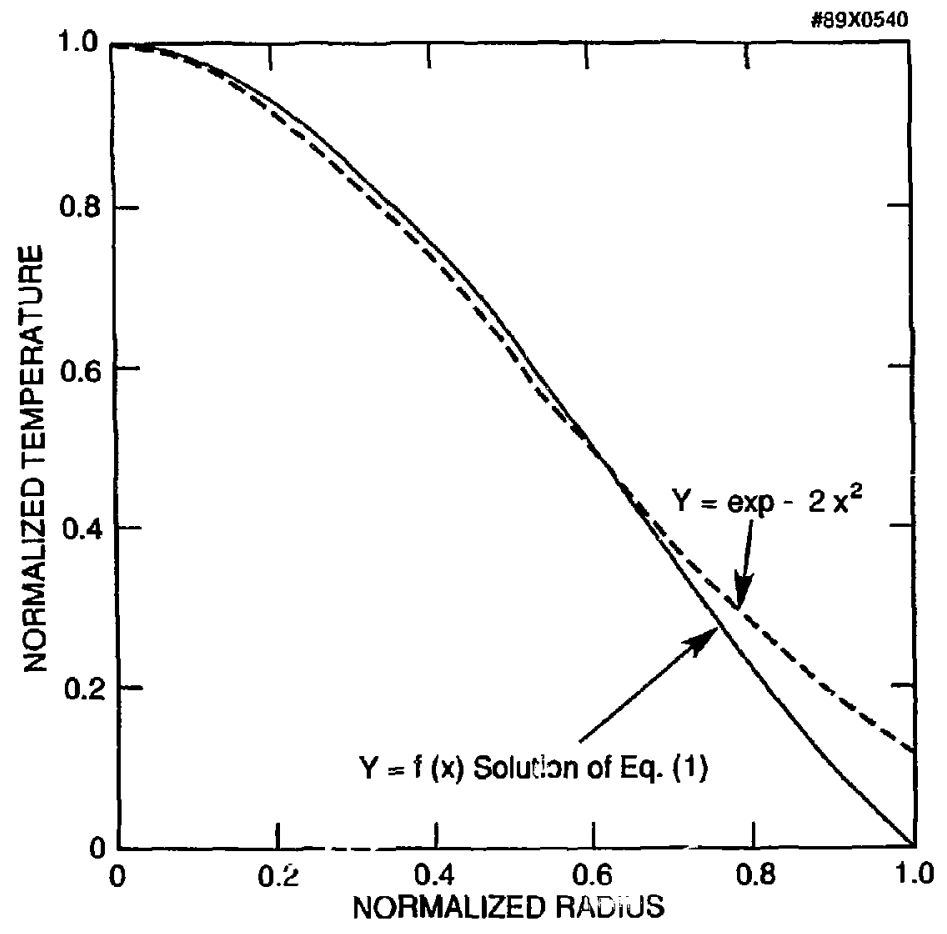

